\title{
Psychotherapy for Phantom Limb Pain
}

\author{
Jiatong $\mathrm{Wei}^{1{ }^{1, *}}$ \\ University of Nottingham, Gatehouse Lodge, Wollaton Hall Drive, Derby Road, Nottingham, NG8 1AF, United \\ Kingdom \\ *Corresponding author. Email: Weijiatong1004@foxmail.com
}

\begin{abstract}
Phantom limb pain is very common among amputees, and up to $80 \%$ of amputees have reported pain-related experiences with phantom limb pain. Various treatment measures have been adopted for PLP in clinical treatment, including psychological treatment actually. The purpose of this article is to review and evaluate psychotherapy that has been proven to be effective in the last 5 years and to understand its development and clinical application. These include hypnotherapy, mirror therapy, virtual reality and augmented reality, motor imagery, eye movement desensitization and reprocessing. A comprehensive literature search was conducted by the authors using PubMed, Google Academic and China National Knowledge Infrastructure, and the search terms "phantom limb pain", "psychological treatment" and "psychological factors" were used to collect all available literature reports on psychological intervention for phantom limb pain, including literature reviews, randomized controlled trials, and case reports. Besides, literature retrieval was also conducted on the mechanisms and applications of relevant psychotherapy. The most commonly used psychotherapy for phantom limb pain is hypnotherapy, mirror therapy, virtual reality and augmented reality technology, motor imagery and eye movement desensitization and reprocessing. These treatments have been proven to be effective in clinical trial studies. However, they still need more clinical randomized controlled trial results to prove its effectiveness and explore the potential mechanism of treatment.
\end{abstract}

Keywords: phantom limb pain, psychological treatment, chronic pain

\section{INTRODUCTION}

Phantom limb pain refers to the feeling that patients can still feel the existence of affected limb and feel the pain or non-pain after amputation. Up to $80 \%$ amputees have reported experiencing phantom limb pain and other pain after amputation [1,2]. Phantom limb pain occurs for different times, but with the time migration, it usually becomes chronic pain, which is different in the nature, frequency and severity of pain [2]. Besides the feeling of pain, phantom limb pain also affects sleep quality, and causes a decrease in patients' life happiness [3]. However, there is still no clear explanation of the pathogenesis so far. In addition, it lacks specific and effective method for the treatment of phantom limb pain in clinic. Hence, the research and treatment of phantom limb pain still need the unremitting efforts of research scholars and medical staff.

Nevertheless, relatively clear pathophysiological mechanisms have been explained. It is divided into peripheral nervous system, central nervous system and psychological factors. In the interpretation of the peripheral nervous system, it can be understood that amputation damages the nerve signal transmission channels related to the missing limb, causing the nerves to be excessively excited and generate electricity spontaneously. At the same time, the changes of central nervous system are also one of the important reasons for phantom limb pain. Central system mechanisms include spinal cord sensitization and cortical reorganization theory [2]. Although psychological factors have not been proved to have a clear correlation with the pathogenesis of phantom limb pain, studies in this field have proved that psychological factors are one of the risk factors for phantom limb pain, including depression, anxiety and stress [3]. From the perspective of risk factor control, psychotherapy can be considered as one of the treatments for phantom limb pain. Therefore, this article mainly discusses the hypnotherapy, mirror therapy, virtual reality and augmented reality, motor imagination, eye movement desensitization and reprocessing psychotherapy techniques. 


\section{HYPNOTHERAPY}

Hypnotherapy has been used in relieving pain for a long time and has a wide range of treatments, especially imagination and suggestion. Hypnotherapy is a kind of psychological intervention that induces patients to enter hypnotic state and then makes therapeutic suggestion so as to turn pain into comfortable feeling or make pain disappear. Some studies have shown that hypnotic analgesia may be related to the regulation of pain-related brain regions and cingulate gyrus. In the research on electroencephalogram, hypnotic analgesia is closely related to high concentration of attention, emotional processing, and cognitive improvement [29]. At the same time, some scholars have proposed that the mechanism of hypnosis to reduce pain may be related to the enhancement of spinal cord inhibition, and this change in spinal cord response can well regulate the bad emotions generated by the pain sensation [30]. Hypnotherapy is recognized as an effective psychological treatment method for phantom limb pain earlier, among which some researchers have shown that hypnotic induction method for phantom limb pain is effective, it can be used as an auxiliary treatment in clinical treatment. At the same time, hypnotic therapy is safe and non-invasive, which can reduce the cost of drug treatment $[4,5]$ and be more easily accepted by patients. However, there are few research literatures on the treatment of phantom limb pain by hypnotherapy alone, most of which are combined with drugs or other treatments, and the existing research literatures also have the problem of small sample size [4-6, Table 1], which makes the effectiveness of hypnotherapy in the treatment of phanto $\mathrm{m}$ limb pain lack more powerful scientific evidence.

Table 1. Literature case summary

\begin{tabular}{|l|l|l|l|}
\hline AUTHOR & DOCUMENT TYPE & CASE LOAD & OUTCOME \\
\hline MACK P [4] & Case report & 1 & Hypnosis induction: effective \\
\hline OAKLEY DA [5] & Case report and review & 2 & Hypnosis can be used as an adjuvant treatment \\
\hline E F SIEGEL[6] & Case report & 1 & The pain is under control \\
\hline
\end{tabular}

\section{MIRROR THERAPY}

Mirror image therapy came into view in 1996, reported by Professor Ramachandran. The patient can see the picture of the healthy side activity through the plane mirror, induce the patient to imagine and enable the affected side to move at the same time, and the patient can continuously stimulate the central regulation system related to phantom limb pain in the human brain through visual feedback, movement observation, imitation and relearning so as to reduce the feeling of phantom limb pain. This is a simple, convenient, fast, and inexpensive treatment that is easier to implement in a hospital. Studies have shown that researchers in many countries have proved that the mirror image therapy is effective and has a good effect on phantom limb pain. In the case of amputation above the elbow reported by Young Kim et al. [7] in South Korea, patients were plagued by phantom limb pain, and the efficacy of other treatments, such as drug therapy and stellate ganglion block, was unsatisfactory. Subsequently, the authors performed mirror image therapy. The patient visited the hospital four times a week, with 15-minute treatment time each time. After one week, one month and three months of treatment, his VAS level dropped to seven-tenths, five-tenths and four-tenths, respectively, and generally remained at four-tenths of VAS, with the drug dosage halved. In addition, Bahar Anaforoulu[8], a Turkish scholar, has compared the efficacy of mirror image therapy and phantom movement in the treatment of phantom limb pain. Through the comparative experiment on 40 lateral tibial amputees, although both treatment methods have achieved good results, the efficacy of mirror image therapy is better than that of phantom movement. Among the existing research data on the effectiveness of mirror image therapy, some scholars believe that it has an impact on patients with short-term phantom limb pain, and there is no clear evidence of a long-term impact. For patients with long-term PLP, mirror image therapy can only be considered as a treatment, but it is not necessarily effective [11]. However, Meltem Yildirim also mentioned that the experience of the patient before the amputation as well as the current personal physical and physiological conditions can affect the effect of mirror therapy [9]. Therefore, it is very important to fully understand the patient's personal situation before treatment. In order to consider the personal situation of patients, sometimes patients cannot go to the hospital for treatment in person due to time conflict, distance and other reasons, sometimes people will consider the remote treatment method, which is challenging for both the therapist and the patient. There are many interfering factors to be excluded, and more studies are needed to prove the efficacy of this method [10]. 


\section{VIRTUAL REALITY AND REALITY AUGMENTATION TECHNOLOGY}

Virtual reality (VR) technology has been gradually applied widely in clinical practice, and has attracted more attention especially in the field of pain management. Virtual reality technology is a kind of computer simulation system of virtual world created artificially. Patients enter the virtual environment through specific devices, make patients feel that they are a part of the virtual environment and interact with the virtual environment. Through different settings, patients are more likely to immerse or participate in it, thus reducing some pain feelings of patients in reality to a certain extent. Moreover, studies have shown that these interactive and immersive virtual reality technologies are more effective than non-immersive technologies, including complex pain syndrome, chronic neck pain, burn, and different degrees of reduction in pain intensity, and it enhances the therapeutic effect of hypnosis in acute pain[12]. Some scholars believe that VR affects pain perception by distracting attention and emotional changes, which can effectively reduce the number of phantom limb pain in patients, reduce the pain severity and discomfort. In the randomized controlled trial of patients with phantom limb pain after unilateral lower extremity amputation written by Wang Peixia and other authors, the number of pain episodes and pain severity in VR group were significantly decreased, and the scores of PRI, VAS and PPI3 were all better than those of the control group[14]. The possible reason may be that the immersive environment created by virtual reality reduces pain sensation by up-regulating non-pain neural signals [13-14]. In addition, augmented reality technology is the original in the real world because of the environment and time constraints of the entity through the computer and other scientific and technological processing, the virtual information (such as lost limbs) applied to reality, perceived by people, so as to achieve the effect of exercise treatment technology [15], in clinical and virtual reality technology and image therapy combined. Although there are multiple research projects that demonstrate the effectiveness of virtual reality technology and augmented reality technology in the treatment of phantom limb pain, due to the small sample size problem in the existing research results, which are mostly case reports, this is not strong enough to support the research results [16]. Therefore, although virtual therapy and augmented reality are increasingly popular among clinical workers and patients, researchers in this field need to spend more time and energy on research and provide more powerful evidence.

\section{MOTOR IMAGINATION}

Motor imagery (MI) is a relatively complex concept, which involves the processes of cognition, sensation and perception. It uses repeated motor imagination without any motor output to activate specific regions of the brain according to motor memory, thus achieving the technology of improving motor performance [17]. Recent studies on the treatment of phantom limb pain and the alleviation of disability degree by hierarchical motor imagination have received the attention of researchers. Unilateral recognition, dominant motor imagination and mirror visual feedback are the treatments of hierarchical motor imagination. Hemilateral recognition, in other words, is the body's ability to distinguish right from left. Patients with phantom limb pain may have unclear left-right distinction [19]. During this phase of training, patients are asked to look at random images of amputations on a computer and use the left or right button to distinguish one side of the body. In the explicit motor imagination stage, patients need to imagine in mind that they are doing the same posture according to the posture on the computer screen, but there is no actual movement [18]. In the final mirror visual feedback stage, the motion image of the healthy side mapped by the plane mirror is projected onto the affected side. The patient can imagine that the affected side is doing the same motion by observing the motion image of the healthy side. The mechanism of graded motor imagery may be that it activates the Somatosensory (S1), Pre-motor and M1 cortices, mirror neurons in the contralateral cerebral hemisphere of the amputated limb [18]. Besides, some scholars have pointed out that graded exercise imagination is more effective than conventional physical therapy in the treatment of phantom limb pain [20]. However, in the findings of a large number of studies, the interference of factors such as the type of amputation, gender, and personal physical condition on the treatment effectiveness has not been considered alone, and there is a lack of more accurate and powerful scientific evidence. Findings regarding whether amputees have impaired motor imagery are inconsistent. Some people have proposed that phantom limb pain is one of the potential factors causing motor imagination disorder after amputation, and some scholars have also proposed that the phantom feeling during the test affected motor imagination [21]. All the above research results need more clear and direct evidence to explain the correlation between phantom limb pain and motor imagination.

\section{EYEBALL MOVEMENT DESENSITIZATION AND REPROCESSING}

Eye movement desensitization and treatment were first used for the treatment of PTSD, and in 1987, psychologist Francine Shapiro[23] discovered by chance that random eye movement can reduce the intensity of negative, distraught thoughts. It then inspired Shapiro to create EMDR psychotherapy. Eye movement 
desensitization and ongoing treatment is a new comprehensive psychotherapy technique [24]. It is widely used in the field of veterans [26] and psychosis [27]. It has achieved very good curative effect in the treatment of PTSD. Besides, research results have shown that EMDR successfully treats the psychological problems of phantom limb pain and amputation [24], and it is a successful psychological treatment, which can be applied to the clinical treatment of phantom limb pain [25]. As for the mechanism of desensitization and reprocessing of eye movement, many scholars have speculated on it, among which the review authors have divided it into three general models: (1) psychological model (2) psychological and physiological model, and (3) neurobiological model [28]. This study indicates that most of the existing studies on the mechanisms of eye movement desensitization and ongoing treatment are speculative to a certain extent, and further determination of the relationship between them and the therapeutic effect is needed. However, although some scholars have shown that the use of EMDR is particularly effective in eliminating pain, and its guided adaptive information processing (AIP) model is consistent with the results of recent neurobiological studies, it still cannot prove the long-term effectiveness of this method for patients with long-term phantom limb pain [27]. In addition, the randomized controlled experimental studies on eyeball movement desensitization and treatment for phantom limb pain are rare so far. Only Rostaminejad et al. [25] conducted follow-up studies in the past five years. In this study, Rostaminejad experimented with EMDR therapy and controlled conditions on 60 lateral lower extremity amputees, where the causes of amputation varied, with trauma accounting for the largest proportion, followed by diabetes-related complications, and cancer for the smallest part. And the time after amputation varies. The control group received routine care, while the patients in the experimental group received 12 times of one-hour EMDR treatment in one month. The patients were required to complete the subjective unit of pain scale and pain rating scale before and after each treatment, and then were followed up after 24 months. The pain intensity of phantom limb pain was decreased in the EMDR group after treatment and at the 24-month follow-up. In contrast, the intensity of phantom limb pain in the control group remained unchanged throughout treatment [26]. Although this study has proved that EMDR can be recommended as a psychological treatment for phantom limb pain, as the time point is still unclear, more precise research evidence is needed to prove the effectiveness of EMDR in the treatment of phantom limb pain.

\section{CONCLUSION}

Phantom limb pain is a complex pain syndrome. Due to the unclear pathogenesis, multi-disciplinary combined treatments such as drugs, physical therapy and psychological treatment are often adopted in clinical practice. Patients with phantom limb pain often have psychological disorders, and psychological factors are one of the risk factors for phantom limb pain, so psychological treatment is very important. The most commonly used psychotherapy for phantom limb pain is hypnosis, mirror therapy, virtual reality and augmented reality technology, motor imagery and eye movement desensitization and reprocessing. These treatments have been proven to be effective in clinical trial studies. However, they still need more clinical randomized controlled trial results to prove its effectiveness and explore the potential mechanism of these treatments.

\section{AUTHORS' CONTRIBUTIONS} Wei.

This paper is independently completed by Jiatong

\section{REFERENCES}

[1] Limakatso K, Bedwell GJ, Madden VJ, Parker R. The prevalence and risk factors for phantom limb pain in people with amputations: A systematic review and meta-analysis. PLoS One. 2020 Oct 14;15(10):e0240431.

doi: 10.1371/journal.pone.0240431. PMID: 33052924; PMCID: PMC7556495.

[2] Hanyu-Deutmeyer AA, Cascella M, Varacallo M. Phantom Limb Pain. 2021 Aug 1. In: StatPearls [Internet]. Treasure Island (FL): StatPearls Publishing; 2021 Jan-. PMID: 28846343.

[3] Fuchs $\quad \mathrm{X}$, Flor H, Bekrater-Bodmann R. Psychological Factors Associated with Phantom Limb Pain: A Review of Recent Findings. Pain Res Manag. 2018 Jun 21;2018:5080123. doi: 10.1155/2018/5080123. PMID: 30057653; PMCID: PMC6051014.

[4] Mack P, Yam AK, Chin AY. Hypnotherapy: a forgotten modality in managing chronic post-traumatic upper limb pain. Ann Acad Med Singap. 2013 Jul, 42 (7): 361-2. PMID: 23949268.

[5] Oakley DA, Whitman LG, Halligan PW. Hypnotic imagery as a treatment for phantom limb pain: two case reports and a review. Clin Rehabil. 2002 Jun;16 (4): 368-77. doi: 10.1191/0269215502cr507oa. PMID: 12061470.

[6] Siegel EF. Control of phantom limb pain by hypnosis. Am J Clin Hypn. 1979 Apr;21(4):285-6. doi: 10.1080/00029157.1979.10403984. PMID: 474456 .

[7] Kim SY, Kim YY. Mirror therapy for phantom limb pain [J]. Korean J Pain, 2012, 25(4):272—274. 
[8] Anaforoğlu Külünkoğlu B, Erbahçeci F, Alkan A. A comparison of the effects of mirror therapy and phantom exercises on phantom limb pain. Turk J Med Sci. 2019 Feb 11;49(1):101-109. doi: 10.3906/sag-1712-166. PMID: 30762318; PMCID: PMC7350828.

[9] Yildirim M, Sen S. Mirror Therapy in the Management of Phantom Limb Pain. Am J Nurs. 2020 Mar;120(3):41-46. doi: 10.1097/01.NAJ.0000656340.69704.9f. PMID: 32079797.

[10] Rothgangel A, Braun S, Smeets R, Beurskens A. Feasibility of a traditional and teletreatment approach to mirror therapy in patients with phantom limb pain: a process evaluation performed alongside a randomized controlled trial. Clin Rehabil. 2019 Oct;33(10):1649-1660. doi: 10.1177/0269215519846539. Epub 2019 May 8. PMID: 31066315.

[11] Xie HM, Zhang KX, Wang S, Wang N, Wang N, Li X, Huang LP. Effectiveness of Mirror Therapy for Phantom Limb Pain: A Systematic Review and Meta-Analysis. Arch Phys Med Rehabil. 2021 Aug 27:S0003-9993(21)01379-4. doi: 10.1016/j.apmr.2021.07.810. Epub ahead of print. PMID: 34461084.

[12] Li A, Montaño Z, Chen VJ, Gold JI. Virtual reality and pain management: current trends and future directions. Pain Manag. 2011 Mar;1(2):147-157. doi: 10.2217/pmt.10.15. PMID: 21779307; PMCID: PMC3138477.

[13] Pourmand A, Davis S, Marchak A, Whiteside T, Sikka N. Virtual Reality as a Clinical Tool for Pain Management. Curr Pain Headache Rep. 2018 Jun 15;22(8):53. doi: 10.1007/s11916-018-0708-2. PMID: 29904806.

[14] Wang Peixia, Li Zhendong. Application and effect observation of virtual reality technology in patients with phantom limb pain after amputation [J]. Journal of Nursing Education, 2020, v.35(08):71-74.

[15] Wang Yongtian, Chen Jing, Cheng Dewen. Introduction to AR Technology: Science Press, 2015.4

[16] Dunn J, Yeo E, Moghaddampour P, Chau B, Humbert S. Virtual and augmented reality in the treatment of phantom limb pain: A literature review. NeuroRehabilitation. 2017;40(4):595-601. doi: 10.3233/NRE-171447. PMID: 28211829.

[17] Dickstein R, Deutsch JE. Motor imagery in physical therapist practice. Phys Ther. 2007
Jul;87(7):942-53. doi: 10.2522/ptj.20060331. Epub 2007 May 1. PMID: 17472948.

[18] Limakatso K, Corten L, Parker R. The effects of graded motor imagery and its components on phantom limb pain and disability in upper and lower limb amputees: a systematic review protocol. Syst Rev. 2016 Sep 1;5(1):145. doi: 10.1186/s13643-016-0322-5. PMID: 27582042; PMCID: PMC5007706.

[19] Nico D, Daprati E, Rigal F, Parsons L, Sirigu A. Left and right hand recognition in upper limb amputees. Brain. 2004;127(1):120-132. doi: 10.1093/brain/awh006. [PubMed] [CrossRef] [Google Scholar]

[20] Limakatso K, Madden VJ, Manie S, Parker R. The effectiveness of graded motor imagery for reducing phantom limb pain in amputees: a randomised controlled trial. Physiotherapy. 2020 Dec;109:65-74. doi: 10.1016/j.physio.2019.06.009. Epub 2019 Jun 28. PMID: 31992445.

[21] Lyu Y, Guo X, Bekrater-Bodmann R, Flor H, Tong $\mathrm{S}$. Phantom limb perception interferes with motor imagery after unilateral upper-limb amputation. Sci Rep. 2016 Feb 16;6:21100. doi: 10.1038/srep21100. PMID: 26879749; PMCID: PMC4754632.

[22] Rostaminejad A, Behnammoghadam M, Rostaminejad M, Behnammoghadam Z, Bashti S. Efficacy of eye movement desensitization and reprocessing on the phantom limb pain of patients with amputations within a 24-month follow-up. Int J Rehabil Res. 2017 Sep;40(3):209-214. doi: 10.1097/MRR.0000000000000227. PMID: 28368869 .

[23] Shapiro, F Eye Movement Desensitization and Reprocessing, Basic Principles, Protocols and Procedures.New York: Guilford. 1995.

[24] Schneider J, Hofmann A, Rost C, Shapiro F. EMDR in the treatment of chronic phantom limb pain. Pain Med. 2008 Jan-Feb;9(1):76-82. doi: 10.1111/j.1526-4637.2007.00299.x. PMID: 18254770

[25] Rostaminejad A, Behnammoghadam M, Rostaminejad M, Behnammoghadam Z, Bashti S. Efficacy of eye movement desensitization and reprocessing on the phantom limb pain of patients with amputations within a 24-month follow-up. Int J Rehabil Res. 2017 Sep;40(3):209-214. doi: 10.1097/MRR.0000000000000227. PMID:28368869.

[26] Silver SM, Rogers S, Russell M. Eye movement desensitization and reprocessing (EMDR) in the 
treatment of war veterans. J Clin Psychol. 2008 Aug;64(8):947-57. doi: 10.1002/jclp.20510. PMID: 18612994.

[27] Adams R, Ohlsen S, Wood E. Eye Movement Desensitization and Reprocessing (EMDR) for the treatment of psychosis: a systematic review. Eur J Psychotraumatol. 2020 Mar 10;11(1):1711349. doi: 10.1080/20008198.2019.1711349. PMID: 32284817 ; PMCID: PMC7144286.

[28] Landin-Romero R, Moreno-Alcazar A, Pagani M, Amann BL. How Does Eye Movement Desensitization and Reprocessing Therapy Work? A Systematic Review on Suggested Mechanisms of Action. Front Psychol. 2018 Aug 13;9:1395. doi: 10.3389/fpsyg.2018.01395. PMID: 30166975; PMCID: PMC6106867.

[29] Yang Lingling. Clinical application of hypnotic analgesia and its neurobiological mechanism [D]. Chongqing Medical University, 2015.

[30] Li Jianwei, Wang Ying. Mechanisms and methods of hypnotic analgesia [J]. Chinese Journal of Pain Medicine, 2009(06):52-53. 